\title{
A NOTE ON DEGREE THEORY FOR GRADIENT MAPPINGS
}

\author{
HERBERT AMANN
}

\begin{abstract}
In this note we give a simple proof for the essentially known fact, that the Leray-Schauder degree of the gradient of a coercive functional on a large ball of a Hilbert space is one. As a simple application we show that the local index of an isolated local minimum of a $C^{1}$-functional on a Hilbert space equals one.
\end{abstract}

1. Let $U$ be an open subset of some real Hilbert space $H$ and suppose that the gradient $\nabla f: U \rightarrow H$ of a given function $f \in C^{\mathbf{1}}(U, \mathbf{R})$ is a compact vector field, that is, $\nabla f=$ id $-F$, where $F \in C(U, H)$ maps bounded sets into compact sets. In this note we give a simple proof for the following useful theorem, where $\mathbf{B}\left(x_{0}, r\right)$ denotes the open ball in $H$, with center $x_{0}$ and radius $r$, and a bar denotes the closure in $H$.

THEOREM. Suppose that, for some $\beta \in \mathbf{R}$, the set $V:=f^{-1}(-\infty, \beta)$ is bounded and $\bar{V} \subset U$. Moreover, suppose that there are numbers $\alpha<\beta$ and $r>0$, and $a$ point $x_{0} \in U$ such that

$$
f^{-1}(-\infty, \alpha] \subset \overline{\mathbf{B}}\left(x_{0}, r\right) \subset V
$$

and

$$
\nabla f(x) \neq 0 \quad \forall x \in f^{-1}[\alpha, \beta] .
$$

Then $\operatorname{deg}(\nabla f, V, 0)=1$.

As easy consequences of this theorem we obtain the following corollaries:

CoRollary 1. Suppose that $U=H$ and $f(x) \rightarrow \infty$ as $\|x\| \rightarrow \infty$. Moreover, suppose that $\nabla f(x) \neq 0$ for $\|x\| \geq r_{0}$ and some $r_{0}>0$. Then there is a number $r_{1} \geq r_{0}$ such that

$$
\operatorname{deg}(\nabla f, \mathbf{B}(0, r), 0)=1 \quad \forall r \geq r_{1} .
$$

Proof. Observe that $f(x)=\left(\|x\|^{2} / 2\right)-\Phi(x)$, where $\nabla \Phi=F$. Since $F$ is compact, $\Phi$ is weakly sequentially continuous (on convex sets) by Vainberg's theorem [10, Theorem 8.2]. Hence $f$ maps bounded sets into bounded sets.

Thus, let $\alpha:=\sup f\left(B\left(0, r_{0}\right)\right)$ and $r_{1}:=\sup \left\{\|x\| \mid x \in f^{-1}(-\infty, \alpha]\right\}$. Moreover, given $r \geq r_{1}$, fix $\beta>\sup f(B(0, r))$. Then the assertion follows from the theorem (with $x_{0}=0$ ) and the excision property of the degree.

In the following corollary we denote by $i\left(\nabla f, x_{0}\right)$ the index of $\nabla f$ at an isolated zero $x_{0}$.

Received by the editors November 16, 1981.

1980 Mathematics Subject Classification. Primary 58E05; Secondary 47H15, 47H10, 34G20.

Key words and phrases. Leray-Schauder degree, critical point theory, nonlinear functional analysis.

() 1982 American Mathematical Society 0002-9939/81/0000-0186/802.25 
COROLlARY 2. Suppose that $x_{0} \in U$ is an isolated critical point of $f$ at which $f$ has a local minimum. Then $i\left(\nabla f, x_{0}\right)=1$.

Proof. We can assume that $x_{0}=0$ and $f(0)=0$, and that $U=\mathbf{B}\left(0, r_{0}\right)$ for some $r_{0}$ such that 0 is the only critical point of $f$. We claim that

$$
\text { inf } f\left(\overline{\mathbf{B}}\left(0, r_{2}\right) \backslash \mathbf{B}\left(0, r_{1}\right)\right)>0
$$

whenever $0<r_{1} \leq r_{2}<r_{0}$. Indeed, otherwise we find $r_{1}, r_{2}$ with $0<r_{1} \leq r_{2}<$ $r_{0}$ and a sequence $\left(x_{k}\right)$ in $\overline{\mathbf{B}}\left(0, r_{2}\right) \backslash \mathbf{B}\left(0, r_{1}\right)$ such that $f\left(x_{k}\right) \rightarrow 0$. We can assume that $\left(x_{k}\right)$ converges weakly to some $x \in \overline{\mathbf{B}}\left(0, r_{2}\right)$. Since $\Phi$ is weakly sequentially continuous, $f$ is weakly sequentially lower semicontinuous. Hence $0 \leq f(x) \leq$ $\varliminf f\left(x_{k}\right)=0$, which implies $x=0$. On the other hand, since $f\left(x_{k}\right) \geq\left(r_{1}^{2} / 2\right)$ 二 $\Phi\left(x_{k}\right)$ and $\Phi\left(x_{k}\right) \rightarrow \Phi(0)=0$, we find $\varliminf f\left(x_{k}\right) \geq r_{1}^{2} / 2$, a contradiction.

Thus, fix $r_{1}$ and $r_{2}$ with $0<r_{1}<r_{2}<r_{0}$ and $\beta:=\inf f\left(\overline{\mathbf{B}}\left(0, r_{2}\right) \backslash \mathbf{B}\left(0, r_{1}\right)\right)$. Then choose $r>0$ such that $\overline{\mathbf{B}}(0, r) \subset f^{-1}(-\infty, \beta)$ and let

$$
\alpha:=2^{-1} \inf f\left(\overline{\mathbf{B}}\left(0, r_{2}\right) \backslash \mathbf{B}(0, r)\right) .
$$

Now the assertion follows from the theorem (with $U=\mathbf{B}\left(0, r_{2}\right)$ ) since $i(\nabla f, 0)=$ $\operatorname{deg}(\nabla f, V, 0)$.

COROLLARY 3. Let the hypotheses of the theorem be satisfied and suppose that $x_{1} \in V$ is a critical point of $f$, which is not a global minimum of $f$ in $V$. Moreover, suppose either that $F$ is differentiable at $x_{1}$ and 1 is not an eigenvalue of the derivative $F^{\prime}\left(x_{1}\right) \in \mathcal{L}(H)$, or that $x_{1}$ is a local minimum. Then $f$ has at least three critical points in $V$.

PROOF. In the first case $F^{\prime}\left(x_{1}\right)$ is a compact linear operator by a well-known theorem of Krasnosel'skii. Thus, since 1 is not an eigenvalue of $F^{\prime}\left(x_{1}\right)$, the derivative of $\nabla f$ at $x_{1}$ is a continuous automorphism of $H$. Hence $x_{1}$ is an isolated zero of $\nabla f$ and $i\left(\nabla f, x_{1}\right)= \pm 1$ by the Leray-Schauder index formula.

Since $f$ is weakly sequentially lower semicontinuous on $\overline{\mathbf{B}}\left(x_{0}, r\right)$, it attains its minimum at some $x_{2} \in \overline{\mathbf{B}}\left(x_{0}, r\right)$. Our assumptions imply that $x_{2}$ is a global minimum of $f$ in $V$ and that $x_{1} \neq x_{2}$. If $x_{1}$ and $x_{2}$ are the only critical points of $f$ in $V$, then by the additivity of the degree, the Theorem, and Corollary 2 , it follows in either case that

$$
1=\operatorname{deg}(\nabla f, V, 0)=i\left(\nabla f, x_{1}\right)+i\left(\nabla f, x_{2}\right)=0 \text { or } 2,
$$

a contradiction.

It seems worthwhile to formulate the following special case of Corollary 3:

Corollary 4. Suppose that $U=H$ and $f(x) \rightarrow \infty$ as $\|x\| \rightarrow \infty$. Moreover, suppose that $x_{1}$ is a critical point of $f$, which is not a global minimum. If either $F$ is differentiable at $x_{1}$ and 1 is not an eigenvalue of the derivative, or $x_{1}$ is a local minimum, then $f$ has at least three critical points.

Proof. This follows by using Corollary 1 instead of the theorem in the proof of Corollary 3.

The results of this paper are known, in principle. To be more precise, Krasnosel'skii [4, Lemma II.6.5] has proven Corollary 1 for the case $H=\mathbf{R}^{n}$ and Thews [9] has extended this result to the general case by approximating $F$ by finite-dimensional 
potential operators and using the standard definition of the Leray-Schauder degree. Corollary 2 has been proven by Rabinowitz [7] under the assumption that $f \in$ $C^{2}(U, \mathbf{R})$. His proof is based on a Ljapunov-Schmidt reduction and on the finitedimensional version of Corollary 2, which has first been derived by Rothe [8] and which, in [7], is deduced from the Poincaré-Hopf theorem. Thews [9], by using the same methods as in his proof of Corollary 1 , has shown that it suffices to assume that $f \in C^{1}(U, \mathbf{R})$. Finally, the arguments based on the additivity of the degree, which are used in the proofs of Corollaries 3 and 4, are standard in degree theory, and more general results of this type can be obtained by Morse theory (cf. [2]).

However, the above results do not seem to be widely known. For example, Castro and Lazer [1] prove (by rather complicated arguments) Corollary 1 for the case $H=\mathbf{R}^{n}$ and under the additional assumption that $f \in C^{2}\left(\mathbf{R}^{n}, \mathbf{R}\right)$ and has only a finite number of critical points. This result has recently been cited by Nirenberg in his survey [6], where he writes: "Though the result seems intuitively clear, I do not know an elementary proof of it." Moreover, Thews's proofs have never been published in a journal. For these reasons the publication of this paper seems to be justified, in particular since we give a short direct proof of the theorem, based upon the ideas of Krasnosel'skii [4], but without finite-dimensional reductions. We use only the homotopy invariance and the normalization property of the LeraySchauder degree and the most basic results about ordinary differential equations. Finally, in some applications it is important to know the value of the degree and the fact that the above results hold for $C^{\mathbf{1}}$ functionals, informations which cannot easily be obtained by other methods.

2. Proof of the theorem. (i) We can assume that $U$ is bounded, so that $F(U)$ is relatively compact. Let $\rho:=\inf \left\{\|\nabla f(x)\| \mid x \in f^{-1}[\alpha, \beta]\right\}$ and observe that $\rho>0$ by the compactness of $F$. By a standard partition of unity argument we can find a (locally) Lipschitz continuous map $G: U \rightarrow H$ such that

$$
\|F(x)-G(x)\| \leq \rho / 2 \quad \forall x \in U
$$

and $G(U) \subset \operatorname{co}(F(U))$, where co denotes the convex hull (e.g. [3, Lemma 1.1]). Hence

$$
g:=\mathrm{id}-G: U \rightarrow H
$$

is a Lipschitz continuous compact vector field such that

$$
(1-t) \nabla f(x)+t g(x)=\nabla f(x)-t(G(x)-F(x)) \neq 0
$$

for $(t, x) \in[0,1] \times \partial V$. Hence, by homotopy invariance,

$$
\operatorname{deg}(\nabla f, V, 0)=\operatorname{deg}(g, V, 0) .
$$

(ii) For each $x \in U$ let $t \rightarrow \varphi^{t}(x)$ be the unique solution of the initial value problem $\dot{y}=-g(y), y(0)=x$, defined on the maximal interval of existence $\left(t^{-}(x), t^{+}(x)\right) \subset \mathbf{R}$. Moreover, let $\Omega:=\left\{(t, x) \in \mathbf{R} \times U \mid t^{-}(x)<t<t^{+}(x)\right\}$ and $\varphi(t, x):=\varphi^{t}(x)$. Then it is well known that $\Omega$ is open in $\mathbf{R} \times U$ and $\varphi: \Omega \rightarrow$ $U$ is Lipschitz continuous. Furthermore, the variation of constants formula implies

$$
\varphi^{t}(x)=e^{-t} x+\int_{0}^{t} e^{-(t-\tau)} G\left(\varphi^{\tau}(x)\right) d \tau=e^{-t}\left[x+K_{t}(x)\right],
$$


where

Finally,

$$
K_{t}(x):=\int_{0}^{t} e^{\tau} G\left(\varphi^{\tau}(x)\right) d \tau \quad \forall(t, x) \in \Omega
$$

$$
\begin{aligned}
f\left(\varphi^{\tau}(x)\right)-f(x) & =\int_{0}^{t}\left[\frac{d}{d \tau} f\left(\varphi^{\tau}(x)\right)\right] d \tau \\
& =-\int_{0}^{t}\left(\nabla f\left(\varphi^{\tau}(x) \mid g\left(\varphi^{\tau}(x)\right)\right) d \tau\right.
\end{aligned}
$$

for $(t, x) \in \Omega$.

Suppose now that $\varphi^{\tau}(x) \in f^{-1}[\alpha, \beta]$ for $0 \leq \tau \leq t$. Then the estimate

$$
\begin{aligned}
(\nabla f(y) \mid g(y)) & =\|\nabla f(y)\|^{2}-(\nabla f(y) \mid G(y)-F(y)) \\
& \geq\|\nabla f(y)\|^{2}-\|\nabla f(y)\| \rho / 2 \geq\|\nabla f(y)\|^{2} / 2 \\
& \geq \rho^{2} / 2 \quad \forall y \in f^{-1}[\alpha, \beta]
\end{aligned}
$$

and (2) imply

$$
\alpha-\beta \leq f\left(\varphi^{t}(x)\right)-f(x) \leq-\rho^{2} t / 2 .
$$

From this and the assumption that $\bar{V} \subset U$ we deduce by standard arguments (e.g. [5, Proposition VI, 1.2]) that $t^{+}(x)=\infty$ for all $x \in \bar{V}$ and that

$$
\varphi^{s}(x) \in f^{-1}(-\infty, \alpha] \quad \forall x \in \partial V,
$$

where $s:=2(\beta-\alpha) \rho^{-2}$.

(iii) For $(t, x) \in(0, s] \times \bar{V}$ let

$$
h_{t}(x):=\left(1-e^{-t}\right)^{-1}\left[x-\varphi^{t}(x)\right]=x-\left(e^{t}-1\right)^{-1} K_{t},
$$

and let $h_{0}:=g$. We claim that $h:[0, s] \times \bar{V} \rightarrow H,(t, x) \rightarrow h_{t}(x)$ is a compact homotopy, that is, $h$ is continuous and $\mathrm{id}_{\bar{V}}-h$ is compact. Indeed, since $\overline{G(\bar{V})}$ is compact, there exists a compact set $C$ such that $e^{\tau} G\left(\varphi^{\tau}(x)\right) \in C$ for $(\tau, x) \in$ $[0, s] \times \bar{V}$. Thus, by approximating the integral by Riemann sums, it follows that

$$
\frac{1}{t} \int_{0}^{t} e^{\tau} G\left(\varphi^{\tau}(x)\right) d \tau \in \overline{\mathrm{co}}(C) \quad \forall(t, x) \in(0, s] \times \bar{V} .
$$

This implies easily that $\mathrm{id}_{\bar{V}}-h$ maps $[0, s] \times \bar{V}$ into a compact set.

The continuity of $h$ on $(0, s] \times \bar{V}$ is obvious. Suppose then that $\left(t_{k}, x_{k}\right)$ is a sequence in $[0, s] \times \bar{V}$ such that $\left(t_{k}, x_{k}\right) \rightarrow(0, x)$. To show that $h_{t_{k}}\left(x_{k}\right) \rightarrow$ $h_{0}(x)$, it suffices to consider the case that $t_{k}>0$ for all $k \in \mathbf{N}$ and to show that $\left(e^{t_{k}}-1\right)^{-1} K_{t_{k}}\left(x_{k}\right) \rightarrow G(x)$. Moreover, since

$$
\left(e^{t}-1\right)^{-1} K_{t}=\left(\frac{e^{t}-1}{t}\right)^{-1} t^{-1} K_{t},
$$

it suffices to show that $t_{k}^{-1} K_{t_{k}}\left(x_{k}\right) \rightarrow G(x)$. Now $M:=\left\{x_{k} \mid k \in \mathbf{N}\right\} \cup\{x\}$ and, consequently, $[0, s] \times M$ and $\overline{\varphi([0, s] \times M)}$ are compact. Since a Lipschitz continuous function is uniformly Lipschitz continuous on compact sets, there exist constants $\lambda$ and $\mu$ such that

$$
\left\|G\left(\varphi^{\tau}\left(x_{k}\right)\right)-G(x)\right\| \leq \lambda\left\|\varphi^{\tau}\left(x_{k}\right)-x\right\| \leq \mu\left\|x_{k}-x\right\|+\lambda\left\|\varphi^{\tau}(x)-x\right\|
$$


for all $\tau \in[0, s]$ and $k \in \mathbf{N}$. From this estimate we deduce that

$$
t_{k}^{-1} K_{t_{k}}\left(x_{k}\right)-G(x)=\frac{1}{t_{k}} \int_{0}^{t_{k}} e^{\tau}\left[G\left(\varphi^{\tau}\left(x_{k}\right)\right)-G(x)\right] d \tau+\left[\frac{e^{t_{k}}-1}{t_{k}}-1\right] G(x) \rightarrow 0
$$

as $k \rightarrow \infty$. Thus $h$ is a compact homotopy.

Since $h_{t}(x) \neq 0$ for all $t \in(0, s]$ by $(3)$, the homotopy invariance of the degree gives

$$
\operatorname{deg}(g, V, 0)=\operatorname{deg}\left(h_{s}, V, 0\right)
$$

(iv) For every $(\sigma, x) \in[0,1] \times \bar{V}$ let

$$
\begin{aligned}
k(\sigma, x): & =\left(1-\sigma e^{-s}\right)^{-1}\left(x-\sigma \varphi^{s}(x)-(1-\sigma) x_{0}\right) \\
& =x-\left(1-\sigma e^{-s}\right)^{-1}\left[\sigma e^{-s} K_{s}(x)+(1-\sigma) x_{0}\right] .
\end{aligned}
$$

Hence $k:[0,1] \times \bar{V} \rightarrow H$ is a compact homotopy such that $k(0, \cdot)=$ id $-x_{0}$ and $k(1, \cdot)=h_{s}$. Clearly, $k(\sigma, x)=0$ iff $x-x_{0}=\sigma\left(\varphi^{s}(x)-x_{0}\right)$. Since (4) implies $\left\|\varphi^{s}(x)-x_{0}\right\| \leq r$ for $x \in \partial V$, and since $\left\|x-x_{0}\right\|>r$ on $\partial V, k(\sigma, x) \neq 0$ for all $(\sigma, x) \in[0,1] \times \partial V$, and, consequently,

$$
\operatorname{deg}\left(h_{s}, V, 0\right)=\operatorname{deg}\left(\mathrm{id}-x_{0}, V, 0\right)=1 .
$$

Now the assertion follows from (1), (5), and (6).

\section{REFERENCES}

1. A. Castro and A. C. Lazer, Critical point theory and the number of solutions of a nonlinear Dirichlet problem, Ann. Mat. Pura Appl. (4) 70 (1979), 113-137.

2. C. C. Chang, Solutions of asymptotically linear operator equations via Morse theory, Comm. Pure Appl. Math. 34 (1981), 693-712.

3. K. Deimling, Ordinary differential equations in Banach spaces, Lecture Notes in Math., vol. 596, Springer-Verlag, Berlin, 1977.

4. M. A. Krasnosel'skii, The operator of translation along the trajectories of differential equations, Transl. Math. Monos., vol. 19, Amer. Math. Soc., Providence, R.I., 1968.

5. R. H. Martin, Nonlinear operators and differential equations in Banach spaces, Wiley, New York, 1976.

6. L. Nirenberg, Variational and topological methods in nonlinear problems, Bull. Amer. Math. Soc. 4 (1981), 267-302.

7. P. H. Rabinowitz, A note on topological degree for potential operators, J. Math. Anal. Appl. 51 (1975), 483-492.

8. E. H. Rothe, $A$ relation between the type numbers of a critical point and the index of the corresponding field of gradient vectors, Math. Nachr. 4 (1950-51), 12-27.

9. K. Thews, Untere Schranken für die Anzahl von Lösungen einer Klasse nichtlinearer Dirichletprobleme, Doctoral Dissertation, Bochum, 1978.

10. M. M. Vainberg, Variational methods for the study of nonlinear operators, Holden-Day, San Francisco, Calif., 1964.

MATHEMATISCHES INSTITUT, UNIVERSITÄT ZÜRICH, RÄMISTRASSE 74, 8001 ZÜRICH, SWITZERLAND 